\title{
INVESTIGACIÓN
}

Recibido: 02/07/2016 --- Aceptado: 10/01/2017 --- Publicado: 15/06/2017

\section{CARACTERÍSTICAS INFLUYENTES EN LA SINIESTRALIDAD LABORAL EN ESTABLECIMIENTOS HOTELEROS DE ANDALUCÍA}

\section{Influential characteristics in workplace of hotel establishments of Andalusia}

Mercedes Raquel García Revilla. Universidad a Distancia de Madrid. España. mercedesraquel.garcia@udima.es

\section{RESUMEN}

El método nos permitió identificar a las variables tamaño de la empresa, nivel de estudios y el espacio para trabajar, como las variables predictoras de siniestralidad. El presente trabajo tiene la finalidad de exponer las variables más predictiva de la siniestralidad en el mundo hotelero de la comunidad de Málaga. La metodología a utilizar es la Regresión Logística tomando como fuente de información la encuesta realizada por UGT Málaga, al conjunto de trabajadores/as del sector turístico de la provincia limitado al estudio del sector hotelero. El principal resultado indica que el tamaño de la empresa, nivel de estudios y el espacio para trabajar de los trabajadores/as poseen un papel predictor de siniestralidad.

\section{PALABRAS CLAVE}

Accidentes laborales - regresión logística - establecimientos - hoteles.

\begin{abstract}
The method allowed us to identify the variables company size, education level and space to work as predictors of accidents.

This paper aims to show the most predictive variables in claims in the hotel world of the city of Malaga. The methodology used is the logistic regression analysis using as source of information the survey by UGT Malaga, to all workers of the tourism sector limited to the study of the hospitality industry province. The main result indicates that company size, education level and work space for workers have a role accident predictor.
\end{abstract}

\section{KEY WORDS}

Accidents at work - logistic regression - establishments - hotels 


\section{CARACTERÍSTICAS INFLUENTES NA SINISTRALIDADE LABORAL EM ESTABELECIMENTOS HOTELEIROS DE ANDALUZIA}

\section{RESUMO}

O método nos permitiu identificar os fatores, tamanho da empresa, nível de estudos e o espaço para trabalhar, como os fatores previsíveis da sinistralidade. O presente trabalho tem a finalidade de expor os fatores mais preditivos da sinistralidade no mundo hoteleiro de Málaga. A metodologia a utilizar e a Regressão Logística tomando como fonte de informação uma pesquisa realizada por U.G.T. Málaga, ao conjunto de trabalhadores do setor turístico da província limitado ao estudo do setor hoteleiro. O principal resultado indica que o tamanho da empresa, nível de estudos e o espaço para trabalhar dos trabalhadores possuem um papel preditor da sinistralidade.

\section{PALAVRAS CHAVE}

Acidentes laborais -Regressão Logística -Estabelecimentos - Hotéis

\section{Cómo citar este artículo}

García Revilla, M. R. (2017). Características influyentes en la siniestralidad laboral en establecimientos hoteleros de Andalucía [Influential characteristics in workplace of hotel establishments of Andalusia]. Vivat Academia. Revista de Comunicación, 139, 33-41. Doi: http://doi.org/10.15178/va.2017.139.33-41 Recuperado de http://www.vivatacademia.net/index.php/vivat/article/view/900

\section{INTRODUCCIÓN}

El turismo es un sector que opera en un entorno cada vez más exigente y más competitivo, lo que le lleva a velar por ofrecer servicios de calidad. Con esta realidad, resulta fundamental cuidar los recursos humanos, el capital humano, puesto que las personas son el activo fundamental de una empresa turística y como tal debe ponerse en valor desarrollando su formación, informándolos, motivándolos, etc.

No obstante, cuando se habla de Recursos Humanos y Calidad de Servicios Prestados a menudo suele olvidarse que también es necesario para ello garantizar un ambiente de trabajo correcto y saludable. Todo ello repercutirá también positivamente en la buena marcha de la organización y, por ende, en la consecución de beneficios.

El bienestar del trabajador puede ser estudiado desde diversos prismas. Si nos quedamos en el estudio del bienestar del trabajador correlacionándolo exclusivamente con el salario, con el sueldo, pecaríamos de reduccionismo. Así, cuestiones como por ejemplo, el bienestar laboral, el clima organizacional son factores que inciden claramente en el bienestar global del trabajador.

Igualmente, la cultura de la seguridad no debe reducirse a los objetivos de ausencia de enfermedades y accidentes. El concepto de seguridad laboral es algo más que cuestiones relacionadas con el entorno físico-técnico del puesto de trabajo. Así 
está formado por varias cuestiones bien diferenciadas: por las condiciones propias e inherentes de la actividad y de la tarea específica que tiene que desarrollar el trabajador, pero también por las relaciones interpersonales que surgen en el puesto de trabajo. Esto quiere decir que, aspectos como el modo de ejercer la dirección, también tendría mucho que ver en este sentido. Por otra parte, es muy importante que el trabajador perciba que desde la dirección y desde la gerencia, se fomenta la salud laboral y la prevención de los riesgos laborales. De esta manera, se considera que una buena política de prevención de riesgos laborales en la organización constituye la clave del éxito.

Saarela (1989) da un paso más y añade las características del propio individuo y las interacciones entre los tres aspectos (técnico, sociales, perfil demográfico). Para este autor estas parcelas son todas claves pues considera que cada accidente de trabajo es un proceso complejo, resultado de riesgos presentes en el medio laboral. Por otra parte, para este autor estos aspectos no son estancos, sino que se encuentran interrelacionados y su desarrollo influye en el buen funcionamiento del resto.

De esta realidad más compleja y amplia es, hace tiempo, consciente la sociedad. Así lo recogen, por ejemplo, los informes de la Agencia Europea para la Seguridad y Salud en el trabajo (2000) en el que se explica la necesidad de desarrollar un sistema metodológico para el control y monitorización de los accidentes laborales en la Unión Europea donde se recojan aspecto que van más allá de los técnicos.

En un principio, la consagración de los derechos relacionados con la seguridad y salud en el trabajo se efectuó con La Declaración Universal de Derechos Humanos de la Asamblea General de la ONU en 1945 (Lanzadera, E. 2002). En esta se recoge un conjunto de principios en los que se consagran los derechos y libertades fundamentales del ser humano, reconocidos por la comunidad internacional y fundamentada en la dignidad e igualdad del género humano.

Sin embargo, a pesar de que para la sociedad es clara la importancia del tema del clima de seguridad y los riesgos laborales y está más que probada su necesidad para el buen desarrollo de los servicios turísticos, desde la óptica de la investigación, este tema presenta algunas lagunas.

El tema estudiado hasta el momento ha sido más fructífero en crear guías y manuales con el propósito de aclarar las normas a seguir por un trabajador del sector para no sufrir un accidente laboral y en estudios descriptivos sobre las ocurrencias de estos sucesos para casos generales. Mientras que en la literatura científica, si bien ha sido un tema notablemente tratado, su estudio se ha centrado más que todo en investigaciones puntuales acerca de cómo factores concretos influyen en estos asuntos por subsectores.

Como se mencionado anteriormente, a través de los años se han venido realizando manuales para la prevención de riesgos laborales en el sector de la hostelería gracias a instituciones relacionadas con el mismo, pero no se ha incursionado mucho en aplicación de técnicas estadísticas para observar los factores que influyen en la ocurrencia de accidentes laborales, específicamente en el sector de la hostelería.

Este trabajo se ha aprovechado de uno de estos escasos estudios descriptivos. En concreto, de la encuesta realizada por UGT Málaga, al conjunto de trabajadores/as del sector turístico de la provincia limitado al estudio al sector hotelero. La muestra definitiva con la que se ha trabajado, eliminadas las referencias 
a empresas no hoteleras, es de 515 encuestas. Dicho trabajo de campo fue la base para un proyecto fruto del convenio de colaboración entre la Universidad de Málaga y dicho sindicato en el que se pretendía describir la situación en cuanto a riesgos laborales en hostelería en esta provincia. Este trabajo resultó ser muy oportuno debido a que proporciona una base de datos completa, coincidente con las variables que habíamos seleccionado en la fase precedente, y todo ello desde el punto de vista del trabajador. Hay que tener en cuenta, que el estudio de los riesgos laborales desde la perspectiva del trabajador/a es clave, pues recoge información en primera persona sobre el tema de siniestralidad y riesgos laborales asociados a sus tareas; y en cuanto a la organización y acciones preventivas emprendidas por la empresa, supone valorarlas desde el sujeto al que van dirigidas. Lo que sin duda refleja de forma más real el ambiente de clima de seguridad laboral.

Sin embargo, aunque los datos proporcionados por esta encuesta han sido la fuente información básica, se han utilizado encuestas a empleados relacionadas con riesgos laborales y entrevistas a responsables de estos menesteres y se han obtenido datos oficiales principalmente del Instituto Nacional de Estadística (INE, 2015) y el Instituto de Estudios Turísticos y Datos para el análisis y difusión de la información estadística del turismo en España (IET, 2015). Anualmente, estos institutos llevan un compendio de los accidentes laborales sufridos en cada sector incluyendo el hotelero, y mediante sus portales web proveen dicha información la cual ha sido útil.

Debemos destacar que para detectar relaciones entre las variables y las influencias conjunta en la ocurrencia de accidentes y enfermedades laborales, tema central de este estudio, son mucho más apropiadas las técnicas multivariables. Estas técnicas tratan de forma conjunta a las variables, sin descuidar el hecho de que interactúan entre ellas, por lo que parece más adecuado inclinarse por técnicas de este tipo. Entre ellas, seleccionamos la regresión logística para alcanzar el objetivo propuesto.

El objetivo de este trabajo consiste en deliberar sobre las variables más predictivas para lo que usamos la regresión logística. En primer lugar se describirá la metodología a utilizar, luego se mostrará el análisis y discusión de resultados, y finalmente se mencionarán principales conclusiones.

\section{METODOLOGÍA}

Con el propósito de obtener el objetivo propuesto, se ha efectuado un estudio de Regresión Logística (para más detalle ver Jovell, 1995 y Ayçaguer, 1994) tomando como fuente de información la encuesta realizada en la comunidad de Málaga.

Con esta metodología se intenta predecir que un empleado con ciertas características, consideradas factores de riesgo, tenga más probabilidad que otro empleado de sufrir un accidente laboral, lo cual puede permitir tomar medidas preventivas a fin de minimizar la presencia de accidentes laborales.

Para ajustar el modelo a estudiar, se seleccionaron las variables determinadas en la encuesta que presentaron asociación significativa con la variable de respuesta o dependiente: Durante el último año ha sufrido algún tipo de accidente en su puesto de trabajo, a través del método de selección stepwise (Steyerberg et. al, 1999). 


\section{ANÁLISIS Y DISCUSIÓN}

El análisis para la determinación de las principales variables que influyen en la siniestralidad laboral, se hace mediante el uso de los datos obtenidos en la Encuesta de UGT y con el uso de la Regresión Logística.

En los pasos del método de selección por stepwise, se llegaron a eliminar las variables: Satisfacción en el entorno laboral, Horas extras, Trayecto de casa a trabajo, Carga física continuadas, Posturas dolorosas o forzadas que implican su trabajo, Cargo con alto grado de responsabilidad, Se lleva los problemas fuera del horario laboral, Ergonomía en el diseño del puesto de trabajo, Mantenimiento o diseño inadecuado o deficiente de las instalaciones, Rapidez, Evaluación de los riesgos laborales en su puesto de trabajo, Formación e información de la empresa en tema de riesgos laborales y Género.

Tras esta primera fase podemos decir que las variables que mejor predicen la posibilidad de tener un accidente laboral fueron Tamaño de la empresa (TAM), Nivel educativo del trabajador (P4) y Falta de espacio en el trabajo (P17_2). Los coeficientes de Wald indican que las tres variables deben ser retenidas ya que aportan significativamente a la predicción de la probabilidad de sufrir accidentes en el puesto de trabajo.

\begin{tabular}{llll} 
& \multicolumn{3}{c}{ Tabla 1. Análisis de los Efectos } \\
Effect & DF & Wald Chi-Square & Pr $>$ ChiSq \\
\hline TAM & 3 & 8.1246 & $0.0435^{*}$ \\
P4 & 4 & 13.1273 & $0.0107^{*}$ \\
P17_2 & 1 & 8.5675 & $0.0034^{* *}$
\end{tabular}

* El efecto es significativamente distinto de cero al nivel 0.05

** El efecto es significativamente distinto de cero al nivel 0.01 .

El modelo estimado resultante se muestra a continuación:

$$
\ln \left[\frac{p(x)}{1-p(x)}\right]=-1.4236+0.504 E M+0.3942 E P-1.4916 E U P+0.4879 P E
$$

Para el modelo, las categorías EM (empresa mediana) y EP (Empresa pequeña) incrementan la probabilidad de que el individuo sufra un accidente laboral, la empresa grande no incrementa el riego de presentar esta condición por lo que no es incluida en el modelo, en los niveles educativos (P4) las categorías analfabeta/primaria, secundaria y FP medio y superior no incrementan ni disminuyen la probabilidad de un accidente laboral, en cambio poseer estudios universitarios o de postgrado (EUP) disminuyen esta probabilidad y, finalmente, que el espacio sea pequeño aumenta o contribuye a la probabilidad de sufrir un accidente 
García Revilla, M. R. Características influyentes en la siniestralidad laboral en establecimientos hoteleros de Andalucía

en el trabajo.

\section{Residual Chi-Square Test}

$\begin{array}{lll}\text { Chi-Square } & \text { DF } & \text { Pr }>\text { ChiSq } \\ 25.9011 & 29 & 0.6308\end{array}$

La prueba de bondad de ajuste Chi-cuadrado afirma que no hay evidencia suficiente para rechazar el modelo planteado $(\chi 2(g l=29)=25.9011 ; p=0.6308)$, lo cual se afirma con una confianza del 99\%. Las pruebas Score, Wald y Ratio de verosimilitud muestran valores de significación asociados al estadístico de prueba $<0.01$, indicando que la hipótesis de nulidad global de los es rechazada, al nivel 0.01 .

\begin{tabular}{llll} 
Test & Chi-Square & DF & Pr $>$ ChiSq \\
\hline Likelihood Ratio & 32.8771 & 8 & $<.0001$ \\
Score & 30.0573 & 8 & 0.0002 \\
Wald & 25.8980 & 8 & 0.0011
\end{tabular}

El test $\chi 2$ de Wald para verificar individualmente el efecto de las variables sobre el riesgo a favor de tener un accidente en el trabajo, indica que para todas las variables incluidas en el modelo el riesgo estimado difiere del valor uno, aumentando o disminuyendo la probabilidad si el signo del parámetro estimado es positivo o negativo respectivamente.

Tabla 2. Tabla de Clasificación

\begin{tabular}{|c|c|c|c|c|c|c|c|c|c|}
\hline $\begin{array}{l}\text { Prob } \\
\text { Level }\end{array}$ & $\begin{array}{l}\text { Correct } \\
\text { Event }\end{array}$ & $\begin{array}{l}\text { Non- } \\
\text { Event }\end{array}$ & $\begin{array}{l}\text { Incorrec } \\
\text { Event }\end{array}$ & $\begin{array}{l}\text { Non- } \\
\text { Event }\end{array}$ & $\begin{array}{l}\text { Percent } \\
\text { Correct }\end{array}$ & $\begin{array}{l}\text { Sensi- } \\
\text { tivity }\end{array}$ & $\begin{array}{l}\text { Speci- } \\
\text { ficity }\end{array}$ & $\begin{array}{l}\text { False } \\
\text { POS }\end{array}$ & $\begin{array}{l}\text { False } \\
\text { NEG }\end{array}$ \\
\hline 0.000 & 61 & 0 & 247 & 0 & 19.8 & 100.0 & 0.0 & 80.2 & . \\
\hline 0.020 & 60 & 7 & 240 & 1 & 21.8 & 98.4 & 2.8 & 80.0 & 12.5 \\
\hline 0.040 & 59 & 15 & 232 & 2 & 24.0 & 96.7 & 6.1 & 79.7 & 11.8 \\
\hline 0.060 & 58 & 55 & 192 & 3 & 36.7 & 95.1 & 22.3 & 76.8 & 5.2 \\
\hline 0.080 & 57 & 60 & 187 & 4 & 38.0 & 93.4 & 24.3 & 76.6 & 6.3 \\
\hline 0.100 & 52 & 74 & 173 & 9 & 40.9 & 85.2 & 30.0 & 76.9 & 10.8 \\
\hline 0.120 & 52 & 92 & 155 & 9 & 46.8 & 85.2 & 37.2 & 74.9 & 8.9 \\
\hline 0.140 & 50 & 104 & 143 & 11 & 50.0 & 82.0 & 42.1 & 74.1 & 9.6 \\
\hline 0.160 & 46 & 118 & 129 & 15 & 53.2 & 75.4 & 47.8 & 73.7 & 11.3 \\
\hline 0.180 & 37 & 137 & 110 & 24 & 56.5 & 60.7 & 55.5 & 74.8 & 14.9 \\
\hline 0.200 & 37 & 157 & 90 & 24 & 63.0 & 60.7 & 63.6 & 70.9 & 13.3 \\
\hline 0.220 & 35 & 163 & 84 & 26 & 64.3 & 57.4 & 66.0 & 70.6 & 13.8 \\
\hline 0.240 & 33 & 166 & 81 & 28 & 64.6 & 54.1 & 67.2 & 71.1 & 14.4 \\
\hline 0.260 & 29 & 171 & 76 & 32 & 64.9 & 47.5 & 69.2 & 72.4 & 15.8 \\
\hline 0.280 & 25 & 182 & 65 & 36 & 67.2 & 41.0 & 73.7 & 72.2 & 16.5 \\
\hline 0.300 & 18 & 205 & 42 & 43 & 72.4 & 29.5 & 83.0 & 70.0 & 17.3 \\
\hline 0.320 & 16 & 222 & 25 & 45 & 77.3 & 26.2 & 89.9 & 61.0 & 16.9 \\
\hline 0.340 & 15 & 228 & 19 & 46 & 78.9 & 24.6 & 92.3 & 55.9 & 16.8 \\
\hline 0.360 & 15 & 230 & 17 & 46 & 79.5 & 24.6 & 93.1 & 53.1 & 16.7 \\
\hline
\end{tabular}


García Revilla, M. R. Características influyentes en la siniestralidad laboral en establecimientos hoteleros de Andalucía

$\begin{array}{llllllllll}0.380 & 15 & 231 & 16 & 46 & 79.9 & 24.6 & 93.5 & 51.6 & 16.6 \\ 0.400 & 15 & 236 & 11 & 46 & 81.5 & 24.6 & 95.5 & 42.3 & 16.3 \\ 0.420 & 15 & 236 & 11 & 46 & 81.5 & 24.6 & 95.5 & 42.3 & 16.3 \\ 0.440 & 15 & 238 & 9 & 46 & 82.1 & 24.6 & 96.4 & 37.5 & 16.2 \\ 0.460 & 9 & 238 & 9 & 52 & 80.2 & 14.8 & 96.4 & 50.0 & 17.9 \\ 0.480 & 8 & 238 & 9 & 53 & 79.9 & 13.1 & 96.4 & 52.9 & 18.2 \\ 0.500 & 3 & 238 & 9 & 58 & 78.2 & 4.9 & 96.4 & 75.0 & 19.6 \\ 0.520 & 0 & 242 & 5 & 61 & 78.6 & 0.0 & 98.0 & 100.0 & 20.1 \\ 0.540 & 0 & 245 & 2 & 61 & 79.5 & 0.0 & 99.2 & 100.0 & 19.9 \\ 0.560 & 0 & 247 & 0 & 61 & 80.2 & 0.0 & 100.0 & . & 19.8\end{array}$

En la tabla de clasificación se puede determinar el punto de corte que permita una mayor sensibilidad o especificidad según el interés del estudio. En este caso debido a la poca información o baja incidencia de accidentes en el trabajo, el modelo sólo permite detectar como máximo el $60,7 \%$ de las personas que sufrirán un accidente en el trabajo, pero indicando en el 74,8\% de las veces que la persona sufrirá un accidente cuando en realidad no lo hará (Falsos positivos) cuando la probabilidad generada por el modelo sea superior a 0.18 (punto de corte).

Si se toma el punto de corte en 0.24, el modelo permitirá identificar correctamente el $67,2 \%$ de las personas que nunca sufrirán un accidente en el trabajo (especificidad o verdaderos negativos) mientras que producirá $71.1 \%$ de falsos positivos.

En general el ajuste del modelo se considera bueno ya que la curva COR (cueva Característica de Operaciones del Receptor) , muestra un área bajo la curva de 0.72 $(<.70)$.

La ecuación de estimación para la probabilidad de sufrir un accidente en el trabajo el último año está dada por:

$p($ paciente $=$ Accidente laboral $)$

$$
=\frac{1}{1+\exp [-(-1.4236+0.504 E M+0.3942 E P-1.4916 E U P+0.4879 P E)]}
$$

Finalmente, el exponencial de cada coeficiente del modelo se interpreta como el odds ratio o riesgo relativo de presentar un accidente laboral cuando las demás categorías y variables del modelo están ausentes, tal como se muestra a continuación.

Wald Confidence Interval for Adjusted Odds Ratios

Effect Unit Estimate95\% Confidence Limits

\begin{tabular}{|c|c|c|c|c|}
\hline $\begin{array}{l}\text { Empresa mediana (entre } 50 \text { y } 250 \text { trabajadores) } \\
\text { vs Micro empresa ( hasta } 10 \text { trabajadores) }\end{array}$ & 1.0000 & 3.403 & 1.266 & 9.147 \\
\hline $\begin{array}{l}\text { Empresa pequeña (de } 10 \text { a } 50 \text { trabajadores) vs } \\
\text { Micro empresa ( hasta } 10 \text { trabajadores) }\end{array}$ & 1.0000 & 3.049 & 1.205 & 7.715 \\
\hline $\begin{array}{l}\text { Estudios universitarios/postgrado vs FP nivel } \\
\text { superior }\end{array}$ & 1.0000 & 0.115 & 0.025 & 0.534 \\
\hline Poco espacio vs espacio suficiente & 1.0000 & 2.653 & 1.380 & 5.100 \\
\hline
\end{tabular}


Como resultado podemos decir que el riesgo que una persona que trabaja en una empresa mediana sufra un accidente laboral es 3.4 veces mayor que si trabajara en una microempresa, lo cual puede variar en el 95\% de los casos entre 1.266 y 9.14 veces.

En las empresas pequeñas sus trabajadores tienen 3 veces mayor posibilidades de sufrir un accidente en el trabajo que los trabajadores de una microempresa, lo cual tiene un intervalo para el verdadero valor del riego que varía entre 1.2 y 7.72 veces.

Un empleado con estudio universitario o de postgrado tiene $1 / 0.115=8.696$ casi 9 veces menos posibilidades de sufrir un accidente en el trabajo que un FP nivel superior, cuyo valor verdadero puede variar entre 1.87 y 40 veces.

Finalmente, una empresa con poco espacio para el desempeño de las actividades de sus trabajadores puede incrementar cerca de tres veces la probabilidad de que un empleado sufra un accidente respecto a las empresas en donde el espacio es adecuado o suficiente, lo cual varía para el $95 \%$ de los casos entre 1.3 y 5.1 veces.

\section{CONCLUSIONES}

Con la Regresión Logística se pretendió identificar las variables más predictivas de siniestralidad. El método nos permitió identificar a las variables tamaño de la empresa, nivel de estudios y el espacio para trabajar, como las variables predictoras de siniestralidad. Podemos apreciar que suponen una variable para cada ámbito de actuación (trabajador y empresa) y otra para el propio desempeño de la tarea. Mientras, que de nuevo, el ambiente laboral no aparece como influyente.

De esta manera, se determinó que el riesgo que una persona que trabaja en una empresa mediana sufra un accidente laboral es mayor que si trabajara en una microempresa. Además, en las empresas pequeñas sus trabajadores tienen 3 veces mayor posibilidades de sufrir un accidente en el trabajo que los trabajadores de una microempresa. Asimismo, un empleado con estudio universitario o de postgrado tiene menor posibilidad de sufrir un accidente en el trabajo que un FP nivel superior.

Por último, se puede decir, que una empresa con poco espacio para el desempeño de las actividades de sus trabajadores puede incrementar cerca de tres veces la probabilidad de que un empleado sufra un accidente respecto a las empresas en donde el espacio es adecuado o suficiente.

\section{REFERENCIAS}

Agencia Europea para la Seguridad y la Salud en el Trabajo. (2015). Recuperado de https://osha.europa.eu/es

Ayçaguer, L. C. S. (1994). Excursión a la regresión logística en ciencias de la salud. Madrid: Ediciones Díaz de Santos.

Instituto de Estudios Turísticos (IET). (2015). Recuperado de: http://www.iet.tourspain.es

Instituto Nacional de Estadística (INE). (2015). Recuperado de: http://www.ine.es 
García Revilla, M. R. Características influyentes en la siniestralidad laboral en establecimientos hoteleros de Andalucía

Jovell, A. J. (1995). Análisis de regresión logística. Madrid: Centro de Investigaciones Sociológicas.

Lanzadera, E. (2002). La prevención de riesgos laborales en el contexto internacional y en la Unión Europea: fuentes. Notas técnicas. Madrid: UDIMA.

Saarela, K.L. (1989). A poster campaign for improving safety on shipyard scaffolds. Journal of Safety Research, 20, 177-185.

Steyerberg, E., Eijkemans M., Habbema J. (1999). Stepwise selection in small data sets: a simulation study of bias in logistic regression analysis. Journal of Clinical Epidemiology, 52, 935-42.

\section{AUTOR}

\section{Mercedes Raquel García Revilla}

Doctora, Profesora de la Universidad a Distancia de Madrid (UDIMA) en el Grado de Empresas y Actividades Turísticas y Directora de los Másteres "Dirección de Empresas Hoteleras" y "Planificación y Gestión Turística.

https://www.researchgate.net/researcher/2113295952_Mercedes_Raquel_Garcia_Revilla 